IJMMS 29:4 (2002) 245-249

PII. S0161171202004180

http://ijmms.hindawi.com

(c) Hindawi Publishing Corp.

\title{
ON THE OSCILLATION OF FIRST-ORDER NEUTRAL DELAY DIFFERENTIAL EQUATIONS WITH REAL COEFFICIENTS
}

\author{
IBRAHIM R. AL-AMRI
}

Received 2 February 1999 and in revised form 18 November 1999

We prove sufficient conditions for the oscillation of all solutions of a scalar first-order neutral delay differential equation $\dot{x}(t)-c \dot{x}(t-\tau)+\sum_{i=1}^{n} p_{i} x\left(t-\sigma_{i}\right)=0$ for all $0<c<1$, $\tau, \sigma_{i}>0$, and $p_{i} \in \mathbb{R}, i=1,2, \ldots, n$.

2000 Mathematics Subject Classification: 34C15, 34K40.

1. Introduction. The theory of neutral delay differential equations presents complications and the results which are true for neutral differential equations may not be true for nonneutral differential equations. Besides its theoretical interest, the study of oscillatory behaviour of solutions of neutral delay differential equations has some importance in applications. Neutral delay differential equations appear in networks containing lossless transmission lines (as in high-speed computers where the lossless transmission lines are used to interconnect switching circuits), in the study of vibrating masses attached to an elastic bar and also in population dynamics (see Gopalsamy [5], Györi and Ladas [7], Driver [4], Hale [8], Brayton and Willoughby [3], Agwo [1], and the references therein).

In fact, Zahariev and Bainnov [11] seems to be the first paper dealing with oscillation of neutral equations. A systematic development of oscillation theory of neutral equations was initiated by Ladas and Sficas [10].

Ladas and Schultz [9] obtained a necessary and sufficient condition for oscillation of all solutions of the neutral delay differential equation

$$
\dot{x}(t)+c \dot{x}(t-\tau)+q x(t-\sigma)=0,
$$

where $c, q, \tau$, and $\sigma$ are real numbers. It was proved that all solutions of (1.1) are oscillatory if and only if the characteristic equation

$$
F(\lambda) \equiv \lambda+c \lambda e^{-\lambda \tau}+q e^{-\lambda \sigma}=0
$$

has no real roots.

Also, it was proved for the scalar first-order neutral delay differential equation

$$
\dot{x}(t)+c \dot{x}(t-\tau)+\sum_{i=1}^{n} p_{i} x\left(t-\sigma_{i}\right)=0,
$$

where $c \in \mathbb{R}, \tau, \sigma_{i} \geq 0$, and $p_{i}>0$ for all $i=1,2, \ldots, n$, that all solutions are oscillatory 
if and only if the characteristic equation

$$
F(\lambda) \equiv \lambda+c \lambda e^{-\lambda \tau}+\sum_{i=1}^{n} p_{i} e^{-\lambda \sigma_{i}}=0
$$

has no real roots. This result was generalized by Arino and Györi in [2] for $p_{i} \in \mathbb{R}$.

In [6], Gopalsamy and Zhang proved that, if

(1) $0<c<1$,

(2) $\tau \geq 0, \sigma>0, p \geq 0$,

(3) $p e \sigma>1-c(1+\tau p /(1-c))$,

then every solution of

$$
\dot{x}(t)-c \dot{x}(t-\tau)+p x(t-\sigma)=0
$$

is oscillatory.

In this paper, we extend the last result for a scalar first-order neutral delay differential equation in the form

$$
\dot{x}(t)-c \dot{x}(t-\tau)+\sum_{i=1}^{n} p_{i} x\left(t-\sigma_{i}\right)=0
$$

for all $0<c<1, \tau, \sigma_{i} \geq 0$, and $p_{i} \in \mathbb{R}, i=1,2, \ldots, n$.

Let $\gamma=\max \left\{t, \sigma_{1}, \sigma_{2}, \ldots, \sigma_{n}\right\}$ and let $t_{1} \geq t_{0}$. By a solution of (1.6) on $\left[t_{1}, \infty\right)$ we mean a function $x(t) \in \mathbf{C}\left(\left[t_{1}-\gamma, t_{1}\right], \mathbb{R}\right)$ such that $x(t)-c x(t-\tau)$ is continuously differentiable and (1.6) is satisfied for $t \geq t_{1}$.

As it is customary, a solution is called oscillatory if it has arbitrarily large zeros and otherwise, it is called nonoscillatory.

2. The main result. Consider (1.6) and assume that $p_{k_{i}} \geq 0$ for all $i=1,2, \ldots, \ell$ and $p_{m_{j}}<0$ for all $j=1,2, \ldots, r$ with $\ell+r=n$. Let $q_{m_{j}}=-p_{m_{j}}, j=1,2, \ldots, r$, then (1.6) takes the form

$$
\dot{x}(t)-c \dot{x}(t-\tau)+\sum_{i=1}^{\ell} p_{k_{i}} x\left(t-\tau_{k_{i}}\right)-\sum_{j=1}^{r} q_{m_{j}} x\left(t-\sigma_{m_{j}}\right)=0
$$

or simply

$$
\dot{x}(t)-c \dot{x}(t-\tau)+\sum_{i=1}^{\ell} p_{i} x\left(t-\tau_{i}\right)-\sum_{j=1}^{r} q_{j} x\left(t-\sigma_{j}\right)=0,
$$

where $0<c<1, \tau, \sigma_{i}, p_{i} \geq 0$, and $\tau_{i}, q_{j}>0$ for all $i=1,2, \ldots, \ell$ and all $j=1,2, \ldots, r$ with $\ell+r=n$.

THEOREM 2.1. Consider the neutral delay differential equation (2.2). If

(i) $\ell p_{i}>\sum_{j=1}^{r} q_{j}$ for all $i=1,2, \ldots, \ell$,

(ii) $\sum_{i=1}^{\ell}\left(1-c-\sum_{j=1}^{r} q_{j}\left(\tau_{i}-\sigma_{j}\right)\right) \geq 0$,

(iii) $(e+c \tau /(1-c)) \sum_{i=1}^{\ell}\left(\ell p_{i}-\sum_{j=1}^{r} q_{j}\right) \tau_{i}>\sum_{i=1}^{\ell}\left((1-c)-\sum_{j=1}^{r} q_{j}\left(\tau_{i}-\sigma_{j}\right)\right)$, then all solutions of (2.2) are oscillatory. 
Proof. The characteristic equation of the neutral delay differential equation (2.2) is

$$
F(\lambda) \equiv \lambda-c \lambda e^{-\lambda \tau}+\sum_{i=1}^{\ell} p_{i} e^{-\lambda \tau_{i}}-\sum_{j=1}^{r} q_{j} e^{-\lambda \sigma_{j}}=0 .
$$

Assume that (2.2) has a nonoscillatory solution, then the characteristic equation (2.3) has a real root $\lambda_{0}$, that is,

$$
F\left(\lambda_{0}\right) \equiv \lambda_{0}-c \lambda_{0} e^{-\lambda_{0} \tau}+\sum_{i=1}^{\ell} p_{i} e^{-\lambda_{0} \tau_{i}}-\sum_{i=1}^{r} q_{j} e^{-\lambda_{0} \sigma_{j}}=0 .
$$

But for all $\lambda \in \mathbb{R}$, one can write

$$
\begin{aligned}
\lambda\left(1-c e^{-\lambda \tau}-\sum_{j=1}^{r} q_{j} e^{-\lambda \sigma_{j}} \int_{0}^{\tau_{i}-\sigma_{j}} e^{-\lambda s} d s\right) \\
=\lambda-\lambda c e^{-\lambda \tau}+\sum_{j=1}^{r} q_{j}\left(e^{-\lambda\left(\tau_{i}-\sigma_{j}\right)}-1\right) e^{-\lambda \sigma_{j}} \\
=\lambda-\lambda c e^{-\lambda \tau}-\sum_{j=1}^{r} q_{j} e^{-\lambda \sigma_{j}}+e^{-\lambda \tau_{i}} \sum_{j=1}^{r} q_{j}
\end{aligned}
$$

for all $i=1,2, \ldots, \ell$ and then

$$
\begin{aligned}
& \sum_{i=1}^{\ell} \lambda\left(1-c e^{-\lambda \tau}-\sum_{j=1}^{r} q_{j} e^{-\lambda \sigma_{j}} \int_{0}^{\tau_{i}-\sigma_{j}} e^{-\lambda s} d s\right) \\
& =\ell\left(\lambda-\lambda c e^{-\lambda \tau}-\sum_{j=1}^{r} q_{j} e^{-\lambda \sigma_{j}}\right)+\left(\sum_{i=1}^{\ell} e^{-\lambda \tau_{i}}\right)\left(\sum_{j=1}^{r} q_{j}\right) .
\end{aligned}
$$

From (2.3) and (2.6), one can write

$$
F(\lambda)=\frac{1}{\ell} \sum_{i=1}^{\ell} \lambda\left(1-c e^{-\lambda \tau}-\sum_{j=1}^{r} q_{j} e^{-\lambda \sigma_{j}} \int_{0}^{\tau_{i}-\sigma_{j}} e^{-\lambda s} d s\right)+\sum_{i=1}^{\ell}\left(p_{i}-\sum_{j=1}^{r} q_{j}\right) e^{-\lambda \tau_{i}}
$$

for all $\lambda \geq 0$, we have

$$
F(\lambda)>\frac{1}{\ell}\left\{\sum_{i=1}^{\ell} \lambda\left(1-c-\sum_{j=1}^{r} q_{j}\left(\tau_{i}-\sigma_{j}\right)\right)+\sum_{i=1}^{\ell}\left(\ell p_{i}-\sum_{j=1}^{r} q_{j}\right) e^{-\lambda \tau_{i}}\right\} .
$$

Since $\ell p_{i}>\sum_{j=1}^{r} q_{j}$ for all $i=1,2, \ldots, \ell$ and $\sum_{i=1}^{\ell}\left(1-c-\sum_{j=1}^{r} q_{j}\left(\tau_{i}-\sigma_{j}\right)\right) \geq 0$, it follows that $F(\lambda)>0$ and consequently $F(\lambda)$ has no positive or zero real roots.

From (2.7), we have

$$
\ell F(\lambda)=\lambda\left\{\sum_{i=1}^{\ell}\left(1-c e^{-\lambda \tau}-\sum_{j=1}^{r} q_{j} e^{-\lambda \sigma_{j}} \int_{0}^{\tau_{i}-\sigma_{j}} e^{-\lambda s} d s\right)+\frac{1}{\lambda} \sum_{i=1}^{\ell}\left(\ell p_{i}-\sum_{j=1}^{r} q_{j}\right) e^{-\lambda \tau_{i}}\right\}
$$


In order that $F(\lambda)$ has no roots for all $\lambda<0$, we prove that $F(\lambda)>0$ for all $\lambda<0$ and consequently it is enough to prove that

$$
\sum_{i=1}^{\ell}\left(1-c e^{-\lambda \tau}-\sum_{j=1}^{r} q_{j} e^{-\lambda \sigma_{j}} \int_{0}^{\tau_{i}-\sigma_{j}} e^{-\lambda s} d s\right)<-\frac{1}{\lambda} \sum_{i=1}^{\ell}\left(\ell p_{i}-\sum_{j=1}^{r} q_{j}\right) e^{-\lambda \tau_{i}}
$$

Assume that $-\lambda=\mu$ and put

$$
\begin{aligned}
f_{1}(\mu) & =\sum_{i=1}^{\ell}\left(1-c e^{\mu \tau}-\sum_{j=1}^{r} q_{j} e^{\mu \sigma_{j}} \int_{0}^{\tau_{i}-\sigma_{j}} e^{\mu s} d s\right), \\
f_{2}(\mu) & =\sum_{i=1}^{\ell}\left(\ell p_{i}-\sum_{j=1}^{r} q_{j}\right) \frac{e^{\mu \tau_{i}}}{\mu} .
\end{aligned}
$$

Since $\ell p_{i}>\sum_{j=1}^{r} q_{j}$ for all $i=1,2, \ldots, \ell$, then $f_{2}(\mu)>e \sum_{i=1}^{\ell}\left(\ell p_{i}-\sum_{j=1}^{r} q_{j}\right) \tau_{i}$. We construct a function $f$ in between $f_{1}$ and $f_{2}$ such that $f_{2}-f>0$ and $f-f_{1}>0$. Assume that $f(\mu)=\ell(1-c-c \mu \tau)-\sum_{i=1}^{\ell} \sum_{j=1}^{r} q_{j}\left(\boldsymbol{T}_{i}-\sigma_{j}\right)$.

Then,

$$
\begin{aligned}
f-f_{1} & =\ell(1-c-c \mu \tau)-\sum_{i=1}^{\ell} \sum_{j=1}^{r} q_{j}\left(\tau_{i}-\sigma_{j}\right)-\sum_{i=1}^{\ell}\left(1-c e^{\mu \tau}-\sum_{j=1}^{r} q_{j} e^{\mu \sigma_{j}} \int_{0}^{\tau_{i}-\sigma_{j}} e^{\mu s} d s\right) \\
& =\sum_{i=1}^{\ell}\left(1-c e^{\mu \tau}-\sum_{j=1}^{r} q_{j} e^{\mu \sigma_{j}} \int_{0}^{\tau_{i}-\sigma_{j}} e^{\mu s} d s\right) \\
& =\sum_{i=1}^{\ell}\left\{c\left(e^{\mu \tau}-1-\mu \tau\right)+\sum_{j=1}^{r} q_{j}\left[e^{\mu \sigma_{j}} \int_{0}^{\tau_{i}-\sigma_{j}} e^{\mu s} d s-\left(\tau_{i}-\sigma_{j}\right)\right]\right\}>0, \quad \forall \mu>0, \\
f_{2}-f & =\sum_{i=1}^{\ell} g_{i}(\mu),
\end{aligned}
$$

where

$$
g_{i}(\mu)=\left\{\ell p_{i}-\sum_{j=1}^{r} q_{j}\right\} \frac{e^{\mu \tau_{i}}}{\mu}-(1-c-c \mu \tau)+\sum_{j=1}^{r} q_{j}\left(\tau_{i}-\sigma_{j}\right), \quad i=1,2, \ldots, \ell .
$$

Since $e^{\mu \tau_{i}} / \mu$ has a minimum value at $\mu=1 / \tau_{i}$, then $e^{\mu \tau_{i}} \geq e \tau_{i}$, for all $\mu>0, i=$ $1,2, \ldots, \ell$. Hence,

$$
\begin{aligned}
\left.g_{i}(\mu)\right|_{\mu=1 / \alpha \tau_{i}} & =\left\{\ell p_{i}-\sum_{j=1}^{r} q_{j}\right\} \alpha \tau_{i} e^{i / \alpha}-\left(1-c-c \frac{\tau}{\alpha T_{i}}\right)+\sum_{j=1}^{r} q_{j}\left(\tau_{i}-\sigma_{j}\right), \quad i=1,2, \ldots, \ell \\
& >\left\{\ell p_{i}-\sum_{j=1}^{r} q_{j}\right\} \alpha \tau_{i}-(1-c), \quad \alpha \geq 1 .
\end{aligned}
$$


For

$$
\alpha>\frac{1-c}{\left(\ell p_{i}-\sum_{j=1}^{r} q_{j}\right) \tau_{i}}, \quad i=1,2, \ldots, \ell
$$

we have, $g_{i}(\mu)>0$ for $\mu \in\left(0,1 / \alpha \tau_{i}\right)$. It follows that $g_{i}(\mu)>0$, for all $\mu \in\left(0,\left(\ell p_{i}-\right.\right.$ $\left.\left.\sum_{j=1}^{r} q_{j}\right) \tau_{i} /(1-c)\right)$. We now consider $\mu \geq\left(\ell p_{i}-\sum_{j=1}^{r} q_{j}\right) \tau_{i} /(1-c)$ and note that

$$
\left[f_{2}-f\right]_{\mu} \geq \sum_{i=1}^{\ell}\left(\ell p_{i}-\sum_{j=1}^{r} q_{j}\right)\left(e+\frac{c \tau}{1-c}\right) \boldsymbol{\tau}_{i}-\sum_{i=1}^{\ell}\left((1-c)-\sum_{j=1}^{r} q_{j}\left(\boldsymbol{\tau}_{i}-\sigma_{j}\right)\right)>0
$$

since,

$$
\left(e+\frac{c \tau}{1-c}\right) \sum_{i=1}^{\ell}\left(\ell p_{i}-\sum_{j=1}^{r} q_{j}\right) \tau_{i}>\sum_{i=1}^{\ell}\left((1-c)-\sum_{j=1}^{r} q_{j}\left(\tau_{i}-\sigma_{j}\right)\right) .
$$

EXAMPLE 2.2. Consider the neutral delay differential equation in the form

$$
\frac{d}{d t}(x(t)-c x(t-2 \pi))+x(t-4 \pi)-x(t-2 \pi)+(1-c) x\left(t-\frac{3 \pi}{2}\right)=0 .
$$

This equation has an oscillatory solution $x(t)=\sin t$ but not all solutions are oscillatory since the sufficient conditions-in Theorem 2.1-are not satisfied. In fact, it has a nonoscillatory solution $x(t)=e^{-\lambda t}, 0.0608314<\lambda<0.0608315$.

\section{REFERENCES}

[1] H. A. Agwo, On the oscillation of delay differential equations with real coefficients, Int. J. Math. Math. Sci. 22 (1999), no. 3, 573-578.

[2] O. Arino and I. Györi, Necessary and sufficient condition for oscillation of a neutral differential system with several delays, J. Differential Equations 81 (1989), no. 1, 98-105.

[3] R. K. Brayton and R. A. Willoughby, On the numerical integration of a symmetric system of difference-differential equations of neutral type, J. Math. Anal. Appl. 18 (1967), 182-189.

[4] R. D. Driver, A mixed neutral system, Nonlinear Anal. 8 (1984), no. 2, 155-158.

[5] K. Gopalsamy, Stability and Oscillations in Delay Differential Equations of Population Dynamics, Kluwer, Dordrecht, 1992.

[6] K. Gopalsamy and B. G. Zhang, Oscillation and nonoscillation in first order neutral differential equations, J. Math. Anal. Appl. 151 (1990), no. 1, 42-57.

[7] I. Györi and G. Ladas, Oscillation Theory of Delay Differential Equations, Oxford Mathematical Monographs, Clarendon Press, New York, 1991.

[8] J. K. Hale, Theory of Functional Differential Equations, Springer-Verlag, New York, 1977.

[9] G. Ladas and S. W. Schults, On oscillations of neutral equations with mixed arguments, Hiroshima Math. J. 19 (1989), no. 2, 409-429.

[10] G. Ladas and Y. G. Sficas, Oscillations of neutral delay differential equations, Canad. Math. Bull. 29 (1986), no. 4, 438-445.

[11] A. I. Zahariev and D. D. Baĭnov, Oscillating properties of the solutions of a class of neutral type functional-differential equations, Bull. Austral. Math. Soc. 22 (1980), no. 3, 365-372.

IBRAHIM R. AL-AMRI: DePARTMENT OF MATHEMATICS, FACULTY OF SCIENCE, KING ABDULAZIZ UNIVERSITY, P.O. BOX 30010, MADINAH MUNAWARAH, SAUDI ARABIA 


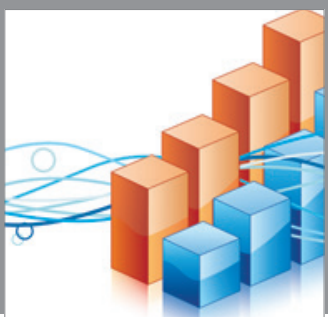

Advances in

Operations Research

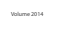

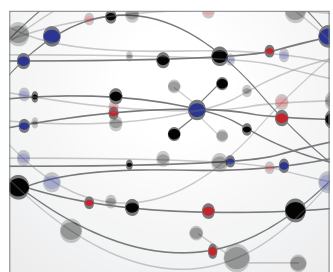

\section{The Scientific} World Journal
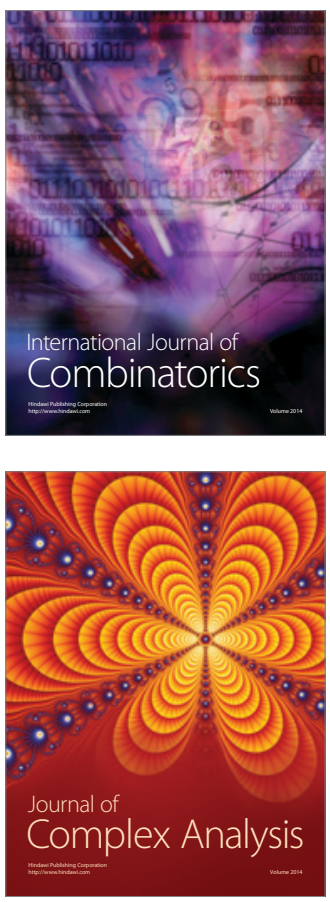

International Journal of

Mathematics and

Mathematical

Sciences
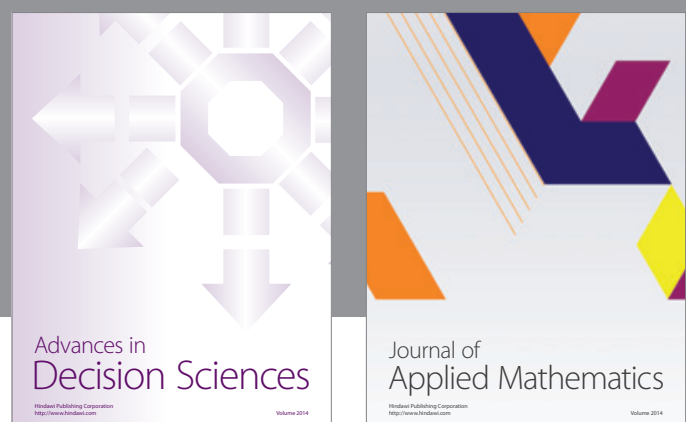

Journal of

Applied Mathematics
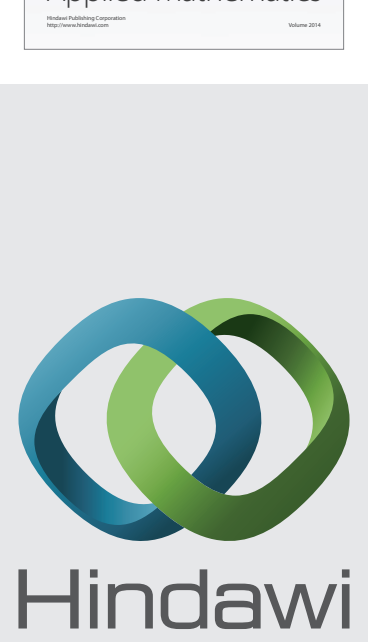

Submit your manuscripts at http://www.hindawi.com
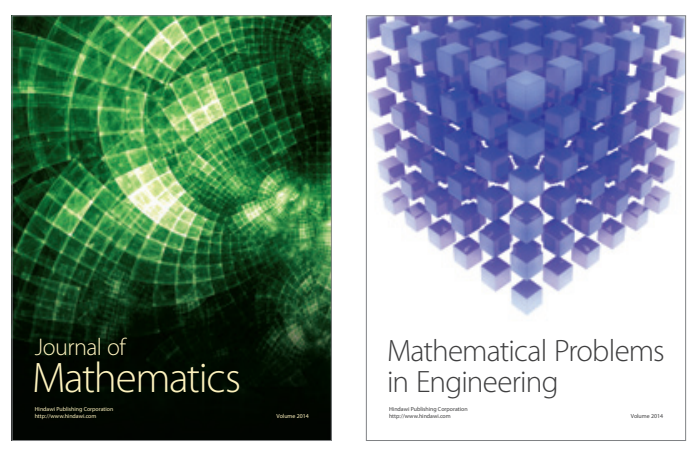

Mathematical Problems in Engineering
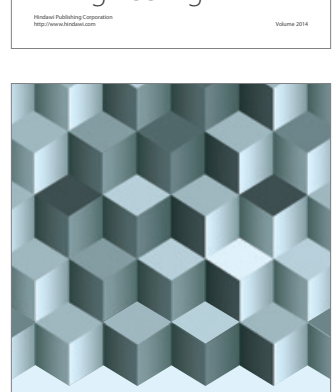

Journal of

Function Spaces
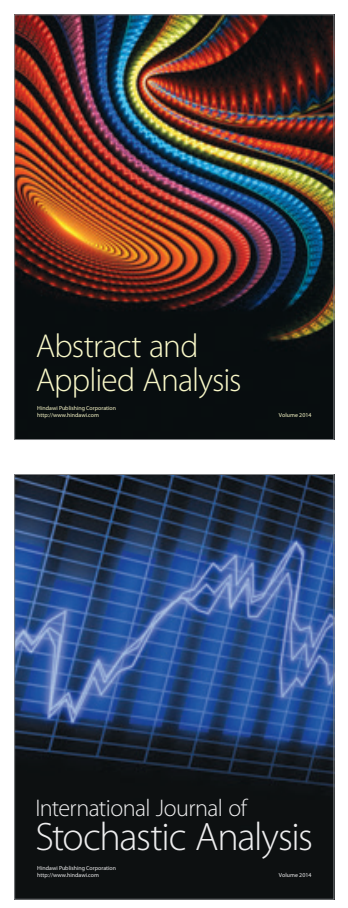

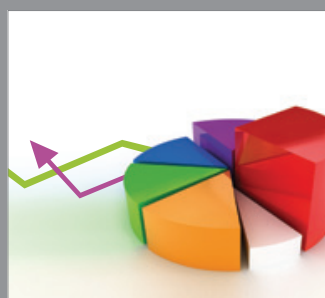

ournal of

Probability and Statistics

Promensencen
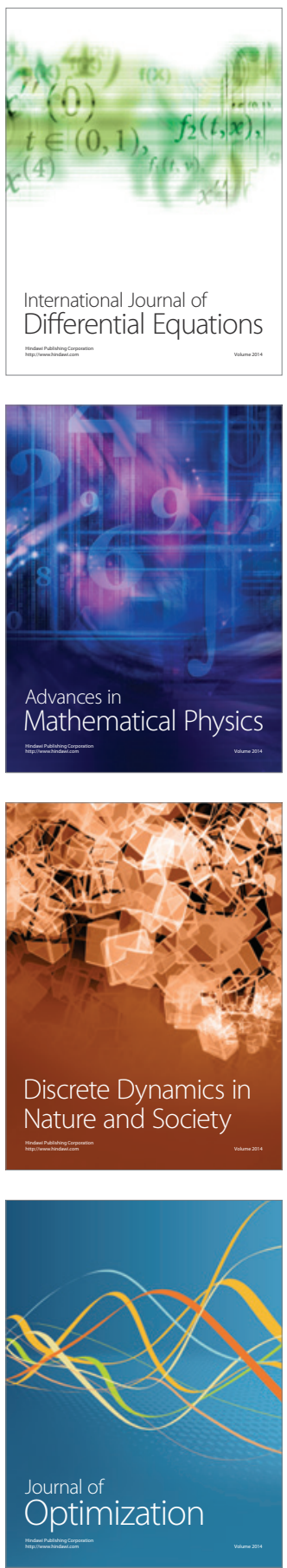